\title{
A Disambiguation Algorithm for Finite Automata and Functional Transducers
}

\author{
Mehryar Mohri \\ Courant Institute of Mathematical Sciences and Google Research \\ 251 Mercer Street, \\ New York, NY 10012, USA
}

\begin{abstract}
We present a new disambiguation algorithm for finite automata and functional finite-state transducers. We give a full description of the algorithm, including a detailed pseudocode and analysis, and several illustrating examples. Our algorithm is often more efficient and the result dramatically smaller than the one obtained using determinization for finite automata or an existing disambiguation algorithm for transducers based on a construction of Schützenberger. In a variety of cases, the size of the unambiguous transducer returned by our algorithm is only linear in that of the input transducer while the transducer given by the construction of Schützenberger is exponentially larger. Our algorithm can be used effectively in many applications to make automata and transducers more efficient to use.
\end{abstract}

\section{Introduction}

Finite automata and transducers are used in a variety of applications in text and speech processing [10,13], bioinformatics [8], image processing [1], optical character recognition [6], and many others. In these applications, automata and transducers are often the result of various complex operations and in general are not efficient to use. Some optimization algorithms such as determinization can make their use more time-efficient. However, the result of determinization is sometimes prohibitively large and not all finite-state transducers are determinizable $[7,11]$.

This paper presents and analyzes an alternative optimization algorithm, disambiguation, which in practice can have efficiency benefits similar to determinization. Our disambiguation algorithm is novel and applies to finite automata, including automata with $\epsilon$-transitions, and to functional finite-state transducers, that is those representing a partial function. Disambiguation returns an automaton or transducer equivalent to the input that is unambiguous, that is one that admits no two accepting paths labeled with the same (input) string. In many instances, the absence of ambiguity can be useful to make search more efficient by reducing the number of paths to explore for very large automata or transducers with several hundred thousand or millions of transitions in text and speech processing or in bioinformatics, and there are many other critical needs for the disambiguation of automata and transducers. 
For finite automata, one way to proceed to obtain an unambiguous and equivalent automaton is simply to apply the standard determinization algorithm. But, as we shall see, for some input automata our algorithm can take exponentially less time than determinization and return an equivalent unambiguous automaton exponentially smaller than the one obtained by using determinization.

For finite-state transducers, disambiguation applies to a broader set of transducers than those that can be determinized using the algorithm described in [11], it applies to any functional transducer. In contrast, it was shown by [3] that a functional transducer is determinizable if and only if it additionally verifies the twins property $[7,11,2]$. Our disambiguation algorithm is also often dramatically more efficient and results in substantially smaller transducers than those obtained using a disambiguation algorithm based on a construction of Schützenberger $[16,15]$, also described by E. Roche and Y. Schabes in the introductory chapter of [14]. In particular, when the input transducer is unambiguous, our algorithm simply returns the same transducer, while the result of the algorithm presented in [14] can be exponentially larger.

The remainder of this paper is organized as follows. In Section 2, we introduce the notation and basic concepts needed for the presentation and analysis of our algorithm. In Section 3, we present our disambiguation algorithm for finite automata in detail, including the proof of its correctness and a brief description of its extension to finite automata with $\epsilon$-transitions. In Section 4, we show how the algorithm can be be used to disambiguate functional transducers and illustrate it with several examples.

\section{Preliminaries}

We will denote by $\epsilon$ the empty string. A finite automaton $A$ with $\epsilon$-transitions is a system $(\Sigma, Q, I, F, E)$ where $\Sigma$ is a finite alphabet, $Q$ a finite set of states, $I \subseteq Q$ the set of initial states, $F \subseteq Q$ the set of final states, and $E$ a finite multiset of transitions, which are elements of $Q \times(\Sigma \cup\{\epsilon\}) \times Q$. We denote by $|A|=|Q|+|E|$ the size of an automaton $A$, that is the sum of the number states and transitions defining $A$.

A path $\pi$ of an automaton is an element of $E^{*}$ with consecutive transitions. The label of a path is the string obtained by concatenation of the labels of its constituent transitions. We denote by $P(p, x, q)$ the set of paths from $p$ to $q$ labeled with $x$ or, more generally, by $P\left(R, x, R^{\prime}\right)$ the set of paths labeled with $x$ from some set of states $R$ to some set of states $R^{\prime}$. We also denote by $P\left(R, R^{\prime}\right)$ the set of all paths from $R$ to $R^{\prime}$. An accepting path is an element of $P(I, F)$. The language accepted by an automaton $A$ is the set of strings labeling its accepting paths and is denoted by $L(A)$. Two automata $A$ and $B$ are said to be equivalent when $L(A)=L(B)$.

We will say that a state $p$ can be reached by a string $x$ when there exists a path from an initial state to $p$ labeled with $x$. When two states can be reached by the same string, we say that they are co-reachable. We will also say that two states $p$ and $q$ share a common future when they admit a common string $x$ to reach a final 
state, that is when there exists a string $x$ such that $P(p, x, F) \cap P(q, x, F) \neq \emptyset$. For any subset $s \subseteq Q$ and $x \in \Sigma^{*}$, we will denote by $\delta(s, x)$ the set of states that can be reached from the states in $s$ by a path labeled with $x$.

A finite-state transducer is a finite automaton in which each transition is augmented with an output label, which is an element of $(\Delta \cup\{\epsilon\})$, where $\Delta$ is a finite alphabet. For any transducer $T$, we denote by $T^{-1}$ its inverse, that is the transducer obtained from $T$ by swapping the input and output label of each transition.

We will use the standard algorithm to compute the intersection $A \cap A^{\prime}$ of two automata $A$ and $A^{\prime}$ [12], whose states are pairs formed by a state of $A$ and a state of $A^{\prime}$, and whose transitions are of the form $\left((p, q), a,\left(p^{\prime}, q^{\prime}\right)\right)$, where $(p, a, q)$ is a transition in $A$ and $\left(p^{\prime}, a, q^{\prime}\right)$ in $A^{\prime}$.

An automaton $A$ is said to be trim if all of its states lie on some accepting path. It is said to be unambiguous if no string $x \in \Sigma^{*}$ labels two distinct accepting paths, finitely ambiguous if there exists $k \in \mathbb{N}$ such that no string labels more than $k$ accepting paths, polynomially ambiguous if there exists a polynomial $P$ with coefficients in $\mathbb{N}$ such that no string $x$ labels more than $P(|x|)$ accepting paths. The finite, polynomial, and exponential ambiguity of an automaton with $\epsilon$-transitions can be tested in polynomial time [4].

\section{Disambiguation algorithm for finite automata}

In this section, we describe in detail our disambiguation algorithm for finite automata. The algorithm is first described for automata without $\epsilon$-transitions. The extension to the case of automata with $\epsilon$-transitions is discussed later. Our algorithm in general does not require a full determinization. In fact, in some cases where the determinization creates $2^{n}$ states where $n$ is the number of states of the input automaton, the cost of our new algorithm or the size of its output is only in $O(n)$.

\subsection{Description}

Figure 1 gives the pseudocode of the algorithm. The first step of the algorithm consists of computing the automaton $A \cap A$ and of trimming it by removing non-coaccessible states (line 1). The cost of this computation is in $O\left(|A|^{2}\right)$ since the complexity of intersection is quadratic and since trimming can be done in linear time. The automaton $B$ thereby constructed can be used to determine in constant time if two states $q$ and $r$ of $A$ that can be reached from $I$ via the same string share a common future simply by checking if $(q, r)$ is a state of $B$. Indeed, by definition of intersection, this property holds iff $(q, r)$ is a state of $B$. As shown by the following proposition, the automaton $B$ is in fact directly related to the ambiguity of $A$.

Proposition 1 ([4]). Let $A$ be a trim finite automaton with no $\epsilon$-transition. A is unambiguous iff no coaccessible state in $A \cap A$ is of the form $(p, q)$ with $p \neq q$. 


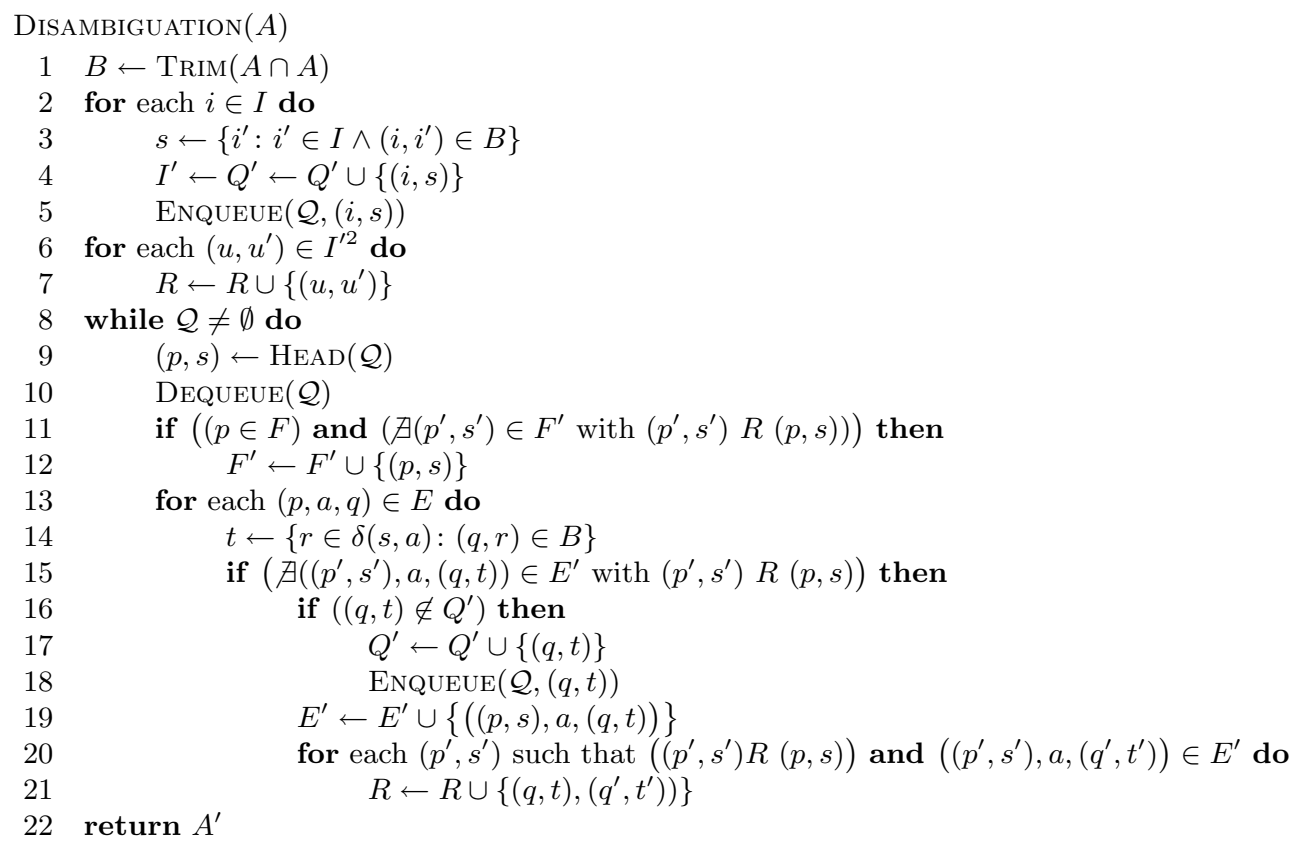

Fig. 1. New disambiguation algorithm for finite automata.

Proof. Since $A$ is trim, the states of $A \cap A$ are all accessible by construction. Thus, a state $(p, q)$ in $A \cap A$ is coaccessible iff it lies on an accepting path, that is by definition of intersection, iff there are two paths $\pi=\pi_{1} \pi_{2} \in P(I, F)$ and $\pi^{\prime}=\pi_{1}^{\prime} \pi_{2}^{\prime} \in P(I, F)$ with $\pi_{1} \in P(I, p)$ and $\pi_{1}^{\prime} \in P(I, q)$, with $\pi_{1}$ and $\pi_{1}^{\prime}$ sharing the same label and $\pi_{2}$ and $\pi_{2}^{\prime}$ also sharing the same label. Thus, $A$ is unambiguous iff $p=q$.

The algorithm constructs an unambiguous automaton $A^{\prime}=\left(Q^{\prime}, E^{\prime}, I^{\prime}, F^{\prime}\right)$. The set of states $Q^{\prime}$ are of the form $(p, s)$ where $p$ is a state of $A$ and $s$ a subset of the states of $A$. Line 2 defines the initial states which are of the form $(i, s)$ with $i \in I$ and $s$ a subset of the states in $I$ sharing a common future with $i$. The algorithm maintains a relation $R$ such that two states of $A^{\prime}$ are in relation via $R$ iff they can be reached by the same string from the initial states. In particular, since all initial states are reachable by $\epsilon$, any two pair of initial states are in relation via $R$ (lines 6-7).

The algorithm also maintains a queue $\mathcal{Q}$ containing the set of states $(p, s)$ of $Q^{\prime}$ left to examine and for which the outgoing transitions are to be determined. The queue discipline, that is the order in which states are added or extracted from $\mathcal{Q}$ is arbitrary and does not affect the correctness of the algorithm. However, different orderings can result in different but equivalent resulting automata.

At each execution of the loop of lines 8-21, a new state $(p, s)$ is extracted from $\mathcal{Q}$ (lines 9-10). To avoid an ambiguity due to finality, state $(p, s)$ is made final only if there is no final state $\left(p^{\prime}, s^{\prime}\right) \in F^{\prime}$ in relation with $(p, s)$ (lines 11-12). 


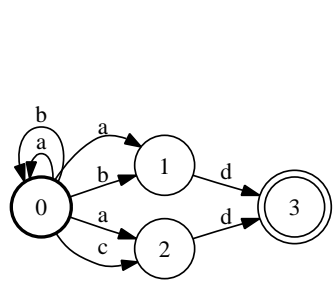

(a)

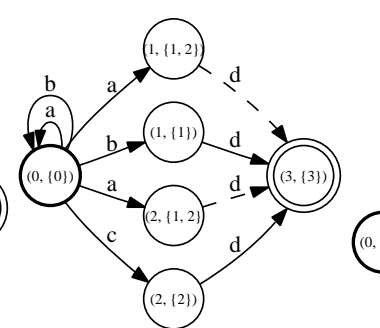

(b)

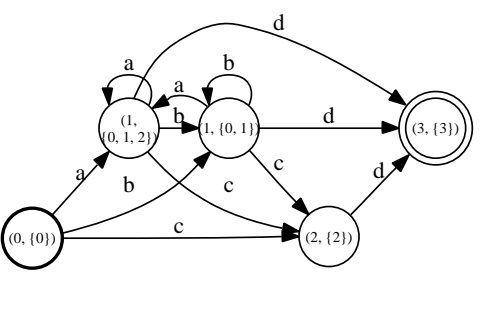

(c)

Fig. 2. Illustration of the disambiguation algorithm. (a) Automaton A. (b) Result of disambiguation algorithm applied to $A$. One of the two dashed transitions is disallowed by the algorithm. (c) Result of determinization applied to $A$.

Each outgoing transition $(p, a, q)$ of $p$ is then examined. Line 14 defines $t$ to be the subset of the states of $A$ that can be reached from a state of $s$ by reading $x$ but excludes states $q^{\prime}$ that do not share a common future with $q$. This is because the subsets are used to detect ambiguities. If $q$ and $q^{\prime}$ do not share a common future even though there are paths with the same label $x$ reaching them, these paths cannot be completed to reach a final state with the same label. Thus, if $X$ is the set of strings leading to a state $(p, s)$ of $Q^{\prime}$, the subset $s$ contains exactly the set of states $r$ of $A$ that can be reached via $X$ from $I$ and that share a common future with $p$.

To avoid creating two paths from $I^{\prime}$ to $(q, t)$ with the same labels, the transition from $(p, s)$ to $(q, t)$ with label $q$ is not created if there exists already one from $\left(p^{\prime}, s^{\prime}\right)$ to $(q, t)$ for a state $\left(p^{\prime}, s^{\prime}\right)$ that can reached by a string also reaching $(p, s)$ (condition of line 15). Note that if $(p, s)$ is extracted from $\mathcal{Q}$ before a state $\left(p^{\prime}, s^{\prime}\right)$ with $\left(p^{\prime}, s^{\prime}\right) R(p, s)$, then the transition from $(p, s)$ to $(q, t)$ is created first and the one from $\left(p^{\prime}, s^{\prime}\right)$ to $(q, t)$ not created. This is how the queue discipline directs the choice of the transitions created.

Lines 16-18 add $(q, t)$ to $Q^{\prime}$ when it is not already in $Q^{\prime}$ and line 19 adds the new transition defined to $E^{\prime}$. After creation of this transition, the destination state $(q, t)$ is then put in relation with all states $\left(q^{\prime}, t^{\prime}\right)$ reached by a transition labeled with $a \in \Sigma$ from a state $\left(p^{\prime}, s^{\prime}\right)$ that is in relation with $(p, s)$.

Figure 2 illustrates the application of the algorithm in a simple case. Observe that states 1 or 2 are not included in the subset of $(0,\{0\})$ in the automaton of Figure 2(b) since 0 does not share a common future with 1 or 2. Figure 2 also shows the result of the application of determinization to the same example. As can be seen from this example, in some instances, determinization creates more transitions than disambiguation. Some states created by the disambiguation algorithm may be non-coaccessible, that is, they may admit no transition to a final state because their output transitions were not constructed to avoid generating ambiguity. These states and the transitions leading to them can be removed in linear time using a standard trimming algorithm. In the case of the 


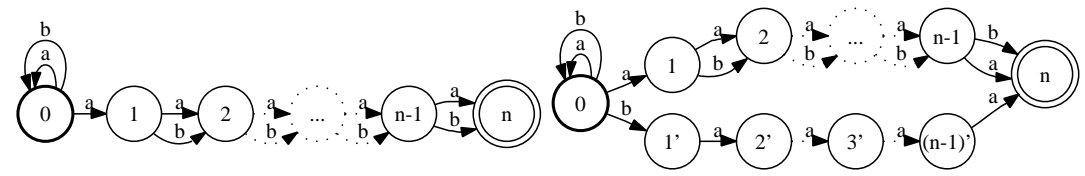

(a)

(b)

Fig. 3. Examples of automata $A$ for which determinization returns an exponentially larger automaton while our algorithm returns $A$ (for (a)) or an automaton whose size is linear in $A$ (for (b)). (a) Automaton representing the regular expression $(a+b)^{*} a(a+b)^{n}$, whose minimal deterministic equivalent has size $\Omega\left(2^{n}\right)$. (b) Automaton representing the regular expression $(a+b)^{*}\left(a(a+b)^{n}+b a^{n}\right)$, whose determinization results in an automaton with $\Omega\left(2^{n}\right)$ states.

automaton of Figure 2(b), the state whose dashed transition is not constructed can be trimmed.

More generally, note that when the input automaton is unambiguous, the subsets created by our algorithm are reduced to singletons: by Proposition 1, a subset cannot contain two distinct states in that case. In such cases, our algorithm simply returns the same automaton $A$. The work done after computation of $B$ is also linear in $|A|$. In contrast, the determinization of $A$ may lead to a blow-up, even when the automaton is unambiguous. In particular, for the standard case of the non-deterministic automaton of Figure 3(a) representing the regular expression $(a+b)^{*} a(a+b)^{n}$, it is known that determinization creates $2^{n+1}-1$ states. However, this automaton is unambiguous and our algorithm returns the same automaton unchanged. The automaton of Figure 3(b) is similar but is ambiguous. Nevertheless, it is not hard to see that again the size of the automaton returned by determinization is exponential and that that of the automaton output by our algorithm is only linear.

\section{$3.2 \quad$ Analysis}

The termination of the algorithm is guaranteed by the fact that the number of states and transitions created must be finite. This is because the number of possible subsets $s$ of states of $A$ is finite, thereby also the number of pairs $(p, s)$ created by the algorithm where $p$ is a state of $A$ and $s$ a subset. Also, the number of transitions created at a state $(p, s)$ is at most equal to the number of states leaving $p$ in $A$. In the worst case, the algorithm may create exponentially many subsets and thus the computational complexity of the algorithm is exponential. In many practical cases, however, this worst case behavior is not observed. In particular, the automaton returned by our disambiguation algorithm is substantially smaller than the one obtained by application of determinization.

We will now show that the automaton returned by the algorithm is unambiguous using the following lemma.

Lemma 1. Let $(q, t)$ and $\left(q^{\prime}, t^{\prime}\right)$ be two states constructed by algorithm DisAMBIGUATION run on input automaton $A$, then $(q, t) R\left(q^{\prime}, t^{\prime}\right)$ iff $(q, t)$ and $\left(q^{\prime}, t^{\prime}\right)$ are co-reachable. 
Proof. We will show by induction on the length of strings $x$ that if two states $(q, t)$ and $\left(q^{\prime}, t^{\prime}\right)$ are both reachable by $x$, then $(p, s) R\left(q^{\prime}, t^{\prime}\right)$. The steps of lines 6-7 ensure that $(q, t) R\left(q^{\prime}, t^{\prime}\right)$ when both states are initial, that is, when they are reachable by $\epsilon$. Assume that it holds for all strings $x$ of length less than or equal to $n$. Let $x=x^{\prime} a$ be a string of length $n+1$ with $x^{\prime} \in \Sigma^{*}$ and $a \in \Sigma$ and assume that $(q, t)$ and $\left(q^{\prime}, t^{\prime}\right)$ are both reachable by $x$. Then, there exists a state $(p, s)$ reachable by $x^{\prime}$ and admitting a transition labeled with $a$ leading to $(q, t)$ and similarly a state $\left(p^{\prime}, s^{\prime}\right)$ reachable by $x^{\prime}$ and admitting a transition labeled with $a$ leading to $\left(q^{\prime}, t^{\prime}\right)$. Then, by the induction hypothesis, we have $(p, s) R\left(p^{\prime}, s^{\prime}\right)$, thus $(q, t) R\left(q^{\prime}, t^{\prime}\right)$ is guaranteed by execution of the steps of lines 20-21. This proves the implication corresponding to one side. The converse holds straightforwardly by construction (lines 6-7 and 20-21).

Proposition 2. The automaton $A^{\prime}$ returned by algorithm DisAmBiguATION run on input automaton $A$ is unambiguous.

Proof. Let $\pi_{1}$ and $\pi_{2}$ be two paths in $A^{\prime}$ from $I^{\prime}$ to $F^{\prime}$ with the same label $x \in \Sigma^{*}$. If $x=\epsilon, \pi_{1}$ is a path from some initial state $\left(i_{1}, s_{1}\right)$ to $\left(i_{1}, s_{1}\right)$ and similarly $\pi_{2}$ a path from some initial state $\left(i_{2}, s_{2}\right)$ to $\left(i_{2}, s_{2}\right)$. All initial states are in relation (lines 6-7), therefore at most one can be made final (lines 11-12). This implies that $\left(i_{1}, s_{1}\right)=\left(i_{2}, s_{2}\right)$ and $\pi_{1}=\pi_{2}$. Let $\left(q_{1}, t_{1}\right)$ be the destination state of $\pi_{1}$ and $\left(q_{2}, t_{2}\right)$ the destination state of $\pi_{2}$. Since $\left(q_{1}, t_{1}\right)$ and $\left(q_{2}, t_{2}\right)$ are both reachable by $x$, by Lemma 1 , we have $\left(q_{1}, t_{1}\right) R\left(q_{2}, t_{2}\right)$. Since no two distinct equivalent states can be made final (lines 11-12), we must have $\left(q_{1}, t_{1}\right)=\left(q_{2}, t_{2}\right)$.

If $x=\epsilon$, this implies that the two paths $\pi_{1}$ and $\pi_{2}$ coincide. If $x \neq \epsilon, x$ can be written as $x=x^{\prime} a$ with $x^{\prime} \in \Sigma^{*}$ and $a \in \Sigma$ and $\pi_{1}$ and $\pi_{2}$ can be decomposed as $\pi_{1}=\pi_{1}^{\prime} e_{1}$ and $\pi_{2}=\pi_{2}^{\prime} e_{2}$ with $e_{1}$ and $e_{2}$ transitions labeled with $a$ leading to $\left(q_{1}, t_{1}\right)$. Let $\left(p_{1}, s_{1}\right)$ be the destination state of $\pi_{1}^{\prime}$ and $\left(p_{2}, s_{2}\right)$ the destination state of $\pi_{2}^{\prime}$. Since $\pi_{1}^{\prime}$ and $\pi_{2}^{\prime}$ are both labeled with $x^{\prime}$, by Lemma 1 , we have $\left(p_{1}, s_{1}\right) R\left(p_{1}^{\prime}, s_{1}^{\prime}\right)$. By the condition of line 15 , if $\left(p_{1}, s_{1}\right) \neq\left(p_{1}^{\prime}, s_{1}^{\prime}\right),\left(p_{1}, s_{1}\right)$ and $\left(p_{1}^{\prime}, s_{1}^{\prime}\right)$ cannot both admit a transition labeled with $a$ and leading to the same state $\left(q_{1}, t_{1}\right)$. Thus, we must have $\left(p_{1}, s_{1}\right)=\left(p_{1}^{\prime}, s_{1}^{\prime}\right)$. Proceeding in the same way with $\pi_{1}^{\prime}$ and $\pi_{2}^{\prime}$ and so on shows that the paths $\pi_{1}$ and $\pi_{2}$ coincide, which concludes the proof.

The following lemmas will be used to show the equivalence between the automaton returned by the algorithm and the input automaton.

Lemma 2. Let $(p, s)$ be a state constructed by algorithm DisAmBiguATION run on input automaton $A$. If $(p, s)$ is reachable by the strings $u$ and $v$ in $A^{\prime}$, then the set of states reachable by $u$ in $A$ and sharing a common future with $p$ coincides with the set of states reachable by $v$ in $A$ and sharing a common future with $p$.

Proof. We show by recurrence on the length of $u$ that if state $(p, s)$ is reachable by $u$ in $A^{\prime}$, then $s$ is the set of states reachable by $u$ and sharing a common future with $p$. This property holds straightforwardly for $u=\epsilon$ by the construction of lines 2-5. Assume now that it holds for all $u$ of length less than or equal to $n$. 
Let $u=u^{\prime} a$ with $u^{\prime} \in \Sigma^{*}$ of length $n$ and $a \in \Sigma$. If $(p, s)$ is reachable by $u$, there must exist some state $\left(p^{\prime}, s^{\prime}\right)$ reachable by $u^{\prime}$ and admitting a transition labeled with $a$ leading to $(p, s)$. By the induction hypothesis, $s^{\prime}$ is the set of states reachable by $u^{\prime}$ and sharing a common future with $p^{\prime}$. By definition of $s$ (line 14), $s=\left\{q \in \delta\left(s^{\prime}, a\right):(q, p) \in B\right\}$, thus the states in $s$ are all reachable by $u$ and share a common future with $p$. Conversely, let $q$ be a state reachable by $u$ and sharing future with $p$. There is a transition labeled with $a$ from some state $q^{\prime}$ reachable by $u^{\prime}$. Since $q^{\prime}$ admits a transition to $q$ labeled with $a$ and $p^{\prime}$ admits a transition labeled with $a$ to $p$, and $p$ and $q$ share a common future, $p^{\prime}$ and $q^{\prime}$ must also share a common future. By the induction hypothesis, $s^{\prime}$ is the set of states reachable by $u^{\prime}$ and sharing a common future with $p^{\prime}$, therefore $q^{\prime}$ is in $s^{\prime}$. Since $q \in \delta\left(q^{\prime}, a\right)$ and $q$ shares a common future with $p$, this implies that $q$ is in $s$. This shows that the states in $s$ are those reachable by $u$ and sharing a common future with $p$.

Lemma 3. Let $A^{\prime}$ be the automaton returned by algorithm DisAmBiguation run on input automaton $A$. Let $q$ be a state reachable in $A$ by string $x$. Then, there exists a state $(q, t)$ in $A^{\prime}$ for some subset $t$ such that $(q, t)$ is reachable by $x$ in $A^{\prime}$.

Proof. We will prove the property by induction on the length of $x$. The property straightforwardly holds for $x=\epsilon$ by the construction steps of lines 2-5. Assume now that it holds for all strings of length less than or equal to $n$ and let $x=u a$ with $u$ a string of length $n$ and $a \in \Sigma$. If $q$ is reachable by string $x$ in $A$, then there exists a state $p_{0}$ in $A$ reachable by $u$ and admitting a transition labeled with $a$ leading to $q$. By the induction hypothesis, there exists a state $\left(p_{0}, s_{0}\right)$ in $A^{\prime}$ reachable by $u$. Now, the property clearly holds for $\left(q, t_{0}\right)$ if the transition labeled with $a$ leaving $\left(p_{0}, s_{0}\right)$ is constructed at lines $15-19$, with $t_{0}$ defined at line 14. Otherwise, by the test of line 15 , there must exist in $A^{\prime}$ a distinct state $\left(p_{1}, s_{0}^{\prime}\right)$ admitting a transition labeled with $a$ leading to $\left(q, t_{0}\right)$ with $\left(p_{1}, s_{0}^{\prime}\right) R\left(p_{0}, s_{0}\right)$. Note that we cannot have $p_{1}=p_{0}$, since the same string cannot reach two distinct states $\left(p_{0}, s_{0}\right)$ and $\left(p_{0}, s_{1}\right)$. Now, since $\left(p_{1}, s_{0}^{\prime}\right)$ admits a transition labeled with $a$ leading to $\left(q, t_{0}\right), p_{1}$ must admit a transition labeled with $a$ and leading to $q$. Thus, $p_{1}$ and $p_{0}$ share a common future in $A$. Since $\left(p_{1}, s_{0}^{\prime}\right) R\left(p_{0}, s_{0}\right)$, by Lemma 1 , they are reachable by a common string $v$. Thus, both $u$ and $v$ reach $\left(p_{0}, s_{0}\right)$. By Lemma 2 , this implies that the set of states in $A$ reachable by $u$ and $v$ and sharing a common future with $p_{0}$ are the same. Since $p_{1}$ and $p_{0}$ share a common future in $A$ and $v$ reaches both $p_{0}$ and $p_{1}, u$ must also reach $p_{1}$ in $A$.

If $u$ reaches $\left(p_{1}, s_{0}^{\prime}\right)$, then $\left(q, t_{0}\right)$ can be reached by $x$ since $\left(p_{1}, s_{0}^{\prime}\right)$ admits a transition labeled with $a$ leading to $(q, t)$. Otherwise, by the induction hypothesis, there must exist a distinct state $\left(p_{1}, s_{1}\right)$ in $A^{\prime}$ reachable by $u$, with $p_{1}$ admitting a transition labeled with $a$ to $q$. Reapplying the argument already presented for $\left(p_{0}, s_{0}\right)$ to $\left(p_{1}, s_{1}\right)$, either we find a path in $A^{\prime}$ labeled with $x$ to a state $\left(q, t_{1}\right)$, or there exists a state $\left(p_{2}, s_{2}\right)$ in $A^{\prime}$ with the same property as $\left(p_{0}, s_{0}\right)$ with $p_{2}$ distinct from $p_{1}$ and $p_{0}$. Since the number of distinct such states is finite, 
reiterating this process guarantees finding a path in $A^{\prime}$ labeled with $x$ to a state $\left(q, t_{k}\right)$ after some finite number of times $k$. Thus, the property holds in all cases.

Lemma 4. Let $A^{\prime}$ be the automaton returned by algorithm Disambiguation run on input automaton $A$, then $L\left(A^{\prime}\right) \subseteq L(A)$.

Proof. The proof argument is similar to that of Lemma 3. Let $x$ be a string reaching a final state $q_{0} \in F$ in $A$. By Lemma 3 , there exists a state $\left(q_{0}, t_{0}\right)$ in $A^{\prime}$ reachable by $x$. If state $\left(q_{0}, t_{0}\right)$ is made final (lines 11-12), this shows that $x$ is accepted by $A^{\prime}$. Otherwise, there must exist a final state $\left(q_{1}, t_{0}^{\prime}\right)$ with $\left(q_{1}, t_{0}^{\prime}\right) R\left(q_{0}, t_{0}\right)$. Note that this implies that $q_{1}$ is final. Note also that we have $q_{1} \neq q_{0}$ since two states $\left(q_{0}, t_{0}\right)$ and $\left(q_{0}, t_{0}^{\prime}\right)$ cannot be co-reachable with $t_{0}^{\prime} \neq t_{0}$. Since $\left(q_{1}, t_{0}^{\prime}\right) R\left(q_{0}, t_{0}\right)$, there exists a string $x_{1}$ reaching both states. Since $\left(q_{0}, t_{0}\right)$ is reachable by both $x$ and $x_{1}$, by Lemma 2 , the set of states in $A$ reachable by $x$ and sharing a common future with $q_{0}$ and those reachable by $x_{1}$ and sharing a common future with $q_{0}$ are the same. $q_{1}$ shares a common future with $q_{0}$ since both states are final and $q_{1}$ is reachable by $x_{1}$, therefore $q_{1}$ is reachable by $x$.

Now, if $x$ reaches $\left(q_{1}, t_{0}^{\prime}\right)$, this shows that $x$ is accepted by $A^{\prime}$. Otherwise, by Lemma 3 , there exists a state $\left(q_{1}, t_{1}\right)$ in $A^{\prime}$ reachable by $x$. We can reapply to $\left(q_{1}, t_{1}\right)$ the same argument as for $\left(q_{0}, t_{0}\right)$ since $q_{1}$ is a final state. Doing so, we either find a final state in $A^{\prime}$ reachable by $x$ or a state $\left(q_{2}, t_{2}\right)$ in $A^{\prime}$ with the same properties as $\left(q_{0}, t_{0}\right)$ with $q_{0}, q_{1}$, and $q_{2}$ all distinct. Since the number of states of $A^{\prime}$ is finite, reiterating this process guarantees finding a final state reachable by $x$. This concludes the proof.

Proposition 3. The automaton $A^{\prime}$ returned by algorithm DisAmBIGUATION run on input automaton $A$ is equivalent to $A$.

Proof. By construction, a path $\left(\left(p_{1}, s_{1}\right), a_{1},\left(p_{2}, s_{2}\right)\right) \cdots\left(\left(p_{k}, s_{k}\right), a_{k},\left(p_{k+1}, s_{k+1}\right)\right)$ is created in $A^{\prime}$ only if the path $\left(p_{1}, a_{1}, p_{2}\right) \cdots\left(p_{k}, a_{k}, p_{k+1}\right)$ exists in $A$, and a state $(p, s)$ is made final in $A^{\prime}$ only if $p$ is final in $A$. Thus, if a string $x=a_{1} \cdots a_{k}$ is accepted by $A^{\prime}$ it is also accepted by $A$, which shows that $L\left(A^{\prime}\right) \subseteq L(A)$. the reverse inclusion holds by Lemma 4 .

The following theorem follows directly by Propositions 2 and 3 .

Theorem 1. The automaton $A^{\prime}$ returned by algorithm Disambiguation run on input automaton $A$ is an unambiguous automaton equivalent to $A$.

Note that the states disallowed via the condition of our algorithm are the minimal ones that can be safely removed from the subsets to check the presence of ambiguities.

\subsection{Disambiguation of automata with $\epsilon$-transitions}

Our algorithm can also be extended to the case of automata with $\epsilon$-transitions. We briefly describe that extension. Let $A$ be an input automaton with $\epsilon$-transitions. 


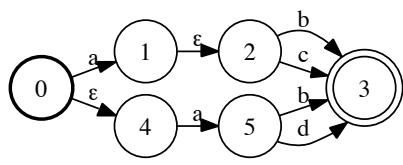

(a)

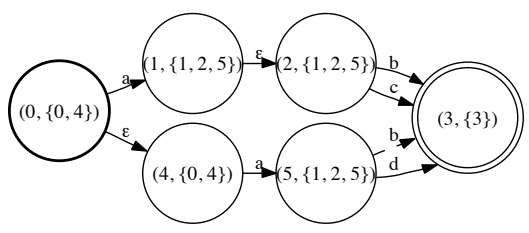

(b)

Fig. 4. (a) Automaton $A$ with $\epsilon$-transitions. (b) Unambiguous automaton equivalent to $A$ returned by our disambiguation algorithm. The dashed transition is disallowed by the algorithm.

Here, the automaton $B$ used to determine pairs of states sharing the same future is obtained similarly by computing the intersection $A \cap A$ by using an $\epsilon$-filter [12] and by trimming the result by removing non-coaccessible states and transitions. For any set $R$ of states of $A$, let $\epsilon[R]$ denote the $\epsilon$-closure of $R$, that is the set of states reachable from states of $R$ via paths labeled with $\epsilon$.

To extend the algorithm to cover the case of automata with $\epsilon$-transitions, it suffices to proceed as follows. The initial states are defined by the set of $(i, s)$ with $i \in I$ and $s=\{q \in \epsilon[I]:(i, q) \in B\}$. At line 14, $\delta(s, a)$ is defined as the set of states reachable from $s$ by reading $a$, including via $\epsilon$-transitions. Finally, the relation $R$ is extended to $\epsilon$-transitions as follows: for each $\left(p^{\prime}, s^{\prime}\right)$ such that $\left(p^{\prime}, s^{\prime}\right) R(p, s)$ and $\left((p, s), \epsilon,\left(q^{\prime}, t^{\prime}\right)\right) \in E^{\prime},\left(p^{\prime}, s^{\prime}\right)$ is put in relation with $\left(q^{\prime}, t^{\prime}\right)$. Figure 4 illustrates the application of our algorithm in that case.

\section{Disambiguation of finite-state transducers}

In this section, we consider the problem of determining an unambiguous transducer equivalent to a given functional finite-state transducer, that is a finite-state transducer representing a (partial) rational function, or equivalently one associating at most one output string to any input string. The functionality of a finite-state transducer $T$ can be tested efficiently from the transducer $T \circ T^{-1}$ as shown by [2].

Theorem 2 ([2]). There exists an algorithm for testing the functionality of a finite-state transducer $T$ with output alphabet $\Delta$ in time $O\left(|E|^{2}+|\Delta||Q|^{2}\right)$.

One possible algorithm for finding an unambiguous transducer equivalent to a functional transducer is determinization [11], however, as discussed earlier, not all functional transducers admit an equivalent deterministic transducer. Figure 5(a) shows an example of such a functional transducer which in fact is unambiguous. A trim functional transducer is determinizable iff it admits the twins property [3].

We will describe instead a disambiguation algorithm does not require that additional property. It is known that any functional transducer can be represented by an unambiguous transducer $[9,5]$. For a functional transducer, by definition, two accepting paths with the same input label have the same output labels. Thus, for disambiguating a functional transducer, only input labels matter and 


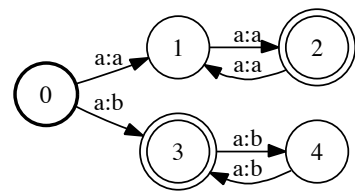

(a)

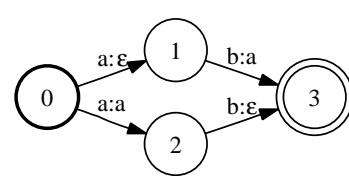

(b)

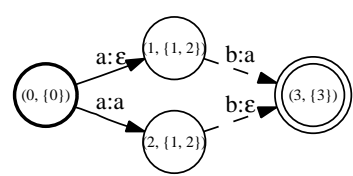

(c)

Fig. 5. (a) Unambiguous finite-state transducer admitting no sequential or deterministic equivalent. (a) Functional transducer T. (b) Disambiguated transducer equivalent to $T$ returned by our algorithm. One of the two dashed transitions is disallowed by the algorithm.

our automata disambiguation can be readily applied to create an unambiguous transducer equivalent to an input functional transducer. Our disambiguation algorithm gives a constructive proof of the existence of an equivalent unambiguous transducer for a rational function. The different possible cross-sections of the construction of [9] correspond to different orders in which transitions are visited and disallowed by our algorithm. Figure 5(b)-(c) illustrates the application of the algorithm in the case of a simple functional transducer.

As already pointed out, our algorithm compares favorably with the existing disambiguation algorithm for finite-state transducers of Schützenberger [16, 15]. That construction can be concisely described as follows. Let $D$ be a deterministic automaton obtained by determinization of the input automaton $A$ of the functional transducer $T$, that is the automaton obtained by removing the output labels of $T$. Then, the algorithm consists of composing $D$ with $T$ using the standard composition algorithm for finite-state transducers while disallowing finality of two composition states $(p, s)$ and $(q, s)$ with the same determinization subset $s$ and distinct states $p$ and $q$ of $T$, and similarly disallowing all but one transition labeled with $a$ from two states $(p, s)$ and $(q, s)$ to the same state, to avoid generating ambiguities. As can be seen from this description, the algorithm requires the determinization of $A$. This is implicit in the description of this construction in [14].

In contrast, our disambiguation algorithm that does not require the determinization of $A$ and as seen in the previous sections can return exponentially smaller automata than those returned by determinization is some cases. Consider for example the finite-state transducers defined as the automata of Figure 3 with each transition augmented with an output label identical to its output label. The construction of Schützenberger requires for those transducers the determinization of the input automata, thus its cost as well as the size of the result are exponential with respect to the size of the output as already discussed in Section 3. Unlike that construction, as in the automata case, our algorithm returns the same transducer or returns one whose size is only linear in that of the input.

The subsets defined by our disambiguation algorithm are never larger than those defined in the subset construction of determinization. This is because for a state $(p, s)$ constructed in the algorithm, only states sharing a common future with $p$ are kept in the subset $s$. In addition to making the size of the subsets shorter, this also reduces the number of states created: two possible states $\left(p, s^{\prime}\right)$ and $\left(p, s^{\prime \prime}\right)$ in the construction of Schützenberger are reduced to the same $(p, s)$ 


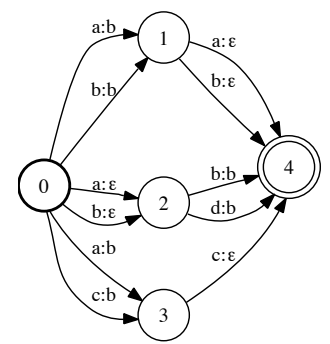

(a)

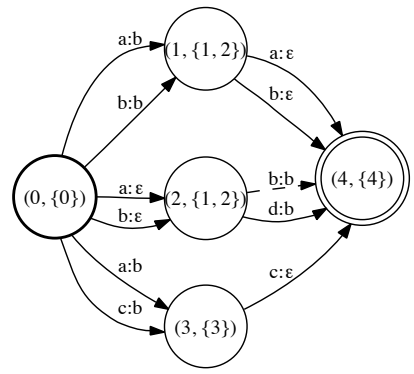

(b)

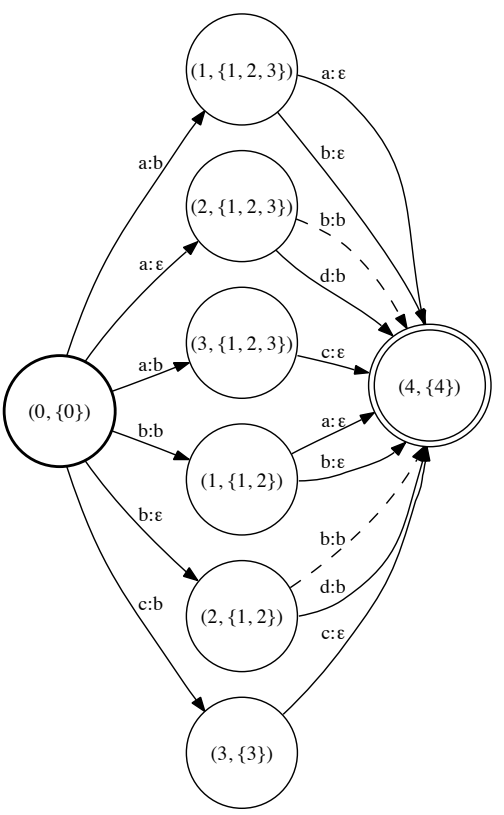

(c)

Fig. 6. Disambiguation of functional transducers. (a) Functional transducer $T$. (b) Unambiguous transducer equivalent to $T$ returned by our algorithm. The dashed transitions are disallowed by the algorithm. (c) Unambiguous transducer returned by the disambiguation construction of Schützenberger [16, 15].

after removal from $s^{\prime}$ and $s$ " of the states not sharing a common future with $p$. This leads in many cases to transducers exponentially smaller than those generated by the construction of Schützenberger and similar improvements in time efficiency.

The observation just emphasized can be illustrated by the simple example of Figure 6. The transducer $T$ of Figure 6(a) is functional but is not unambiguous. Figure $6(\mathrm{~b})$ shows the result of our disambiguation algorithm which is an unambiguous transducer equivalent to $T$ with the same number of states. In contrast, the transducer created by the construction of Schützenberger (Figure 6(c)) has several more states and transitions and some larger subsets.

\section{Conclusion}

We presented a new and often more efficient algorithm for the disambiguation of finite automata and functional transducers. This algorithm is of great practical importance in a variety of applications including text and speech processing, bioinformatics, and in many other applications where they can be used to increase search efficiency. We have also designed a natural extension of these algorithms to some broad families of weighted automata and transducers defined over different semirings. We will present these extensions as well as their theoretical analysis in a longer version of this paper. 


\section{Acknowledgments}

I thank Cyril Allauzen and Michael Riley for discussions about this work. This research was supported by a Google Research Award.

\section{References}

1. J. Albert and J. Kari. Digital image compression. In Handbook of weighted automata. Springer, 2009.

2. C. Allauzen and M. Mohri. Efficient algorithms for testing the twins property. Journal of Automata, Languages and Combinatorics, 8(2):117-144, 2003.

3. C. Allauzen and M. Mohri. Finitely subsequential transducers. International Journal of Foundations of Computer Science, 14(6):983-994, 2003.

4. C. Allauzen, M. Mohri, and A. Rastogi. General algorithms for testing the ambiguity of finite automata and the double-tape ambiguity of finite-state transducers. Int. J. Found. Comput. Sci., 22(4):883-904, 2011.

5. J. Berstel. Transductions and Context-Free Languages. Teubner Studienbucher, 1979.

6. T. M. Breuel. The OCRopus open source OCR system. In Proceedings of ISET/SPIE 20th Annual Symposium, 2008.

7. C. Choffrut. Contributions à l'étude de quelques familles remarquables de fonctions rationnelles. PhD thesis, Université Paris 7, LITP: Paris, France, 1978.

8. R. Durbin, S. R. Eddy, A. Krogh, and G. J. Mitchison. Biological Sequence Analysis: Probabilistic Models of Proteins and Nucleic Acids. Cambridge University Press, 1998.

9. S. Eilenberg. Automata, Languages and Machines, volume A. Academic Press, 1974.

10. R. M. Kaplan and M. Kay. Regular models of phonological rule systems. Computational Linguistics, 20(3), 1994.

11. M. Mohri. Finite-state transducers in language and speech processing. Computational Linguistics, 23(2):269-311, 1997.

12. M. Mohri. Weighted automata algorithms. In Handbook of Weighted Automata, pages 213-254. Springer, 2009.

13. M. Mohri, F. C. N. Pereira, and M. Riley. Speech recognition with weighted finitestate transducers. In Handbook on speech processing and speech communication. Springer, 2008.

14. E. Roche and Y. Schabes, editors. Finite-State Language Processing. MIT Press, 1997.

15. J. Sakarovitch. A construction on finite automata that has remained hidden. Theor. Comput. Sci., 204(1-2):205-231, 1998.

16. M. P. Schützenberger. Sur les relations rationnelles entre monoides libres. Theor. Comput. Sci., 3(2):243-259, 1976. 